\title{
Mid-term follow-up of patients with permanent sequel due to spinal cord ischemia after advanced endovascular therapy for extensive aortic disease
}

\author{
I Mehmedagic ${ }^{1}, S$ Jörgensen ${ }^{2,3}$ and S Acosta ${ }^{1}$
}

Study design: Observational study.

Objectives: Thoraco-abdominal endovascular aortic aneurysm repair (TAEVAR) can be used to treat patients with extensive and complex aortic disease, however, at the risk of spinal cord ischemia (SCI). The aim of this follow-up study was to evaluate the life satisfaction in patients with $\mathrm{SCl}$ after TAEVAR.

Setting: Among 83 patients undergoing TAEVAR between 2009 and 2012 at the Vascular Centre, Malmö, Sweden, 29 developed SCI in-hospital and at follow-up (median 26 months), eight had died and three had no complaints.

Methods: Patients diagnosed with permanent $(n=10)$ and transient $(n=8) \mathrm{SCl}$ were interviewed at home. The Life Satisfaction Questionnaire (LiSat-11) and the Satisfaction With Life Scale (SWLS) were compared with reference samples.

Results: Mid-term mortality in patients with permanent $\mathrm{SCl}(7 / 17)$ was higher than those with transient $\mathrm{SCl}(1 / 12)(P=0.035)$. Ten patients had permanent T1-S5 SCI, two were classified as ASIA Impairment Scale (AIS) A, one as AIS B and seven as AIS D at hospital discharge. Patients diagnosed with transient SCl had residual neurological deficits in the legs $(n=8)$, urge incontinence $(n=3)$ and fecal leakage $(n=2)$ at follow-up. Patients with SCI had lower self-rated life satisfaction in terms of 'life as a whole', 'sexual life', 'somatic health' and 'psychological health' but better in the 'economy' domain.

Conclusion: Assessment of life satisfaction at mid-term follow-up suggests that all patients with SCl in-hospital, whether permanent or transient, should have a multi-disciplinary follow-up regime. Most patients diagnosed with transient neurological deficits had an overlooked permanent, less severe, $\mathrm{SCl}$.

Spinal Cord (2015) 53, 232-237; doi:10.1038/sc.2014.163; published online 23 September 2014

\section{INTRODUCTION}

Patients with complex aortic disease, aneurysm and dissection, live with the risk of aortic rupture, if the disease is left untreated. Today, thoraco-abdominal endovascular aortic aneurysm repair (TAEVAR) is used to treat these patients. However, with this technique, there is a risk of coverage of the vital arteries that supply the spinal cord, which may lead to spinal cord ischemia (SCI) and paraplegia. The incidence of SCI after thoracic endovascular repair has been estimated to be around $3-10 \%,{ }^{1-3}$ whereas the patients with more advanced aortic disease, such as those with thoraco-abdominal aneurysms in need of a more extensive coverage of the aorta, have a higher subsequent risk of SCI after TAEVAR. ${ }^{4}$ The risk of rupture in patients with asymptomatic aortic aneurysm is mainly assessed by maximal diameter of the aneurysm and/or growth of the aneurysm and should be carefully balanced against risk of perioperative morbidity and mortality and expected remaining life-time due to comorbidity factors such as cardio-pulmonary disease. ${ }^{5}$

One artery often described in these contexts is the artery of Adamkiewicz. Anatomical variations of the origin of this artery are common. In the majority of people it originates between Th8 and L1, but can span from Th7 to L3, supplying the lower two-thirds of the spinal cord via the anterior spinal artery. When damaged, it can result in anterior spinal cord syndrome manifested by paraplegia and sensory loss of pain and temperature. ${ }^{6}$

Paraplegia should be considered as one of the worst possible complications to TAEVAR, and knowledge of these patients after discharge in terms of survival, improvement of neurological deficiencies and quality of life is most important to add in the preoperative information before any surgery. The aim of this study on highly selected patients with extensive aortic disease in need of extensive stentgraft therapy of the aorta was to follow up those patients with permanent sequel due to SCI after TAEVAR to evaluate their daily life situation and life satisfaction some years after onset of SCI.

\section{MATERIALS AND METHODS}

\section{Patients}

Eighty-three patients at high risk for SCI underwent advanced endovascular therapy and cerebro-spinal fluid drain insertion for complex aortic disease at the Vascular Center, Malmö, Skåne University Hospital, Sweden, between 2009 and 2012. Twenty-nine patients suffered from SCI perioperatively, ${ }^{7}$ twenty-two had cerebrospinal fluid drains inserted prophylactically and nine postoperatively due to diagnosis of SCI. Transient SCI was defined as full recovery of neurological deficits in-hospital according to medical records. Seventeen and

${ }^{1}$ Vascular Center, Institution for Clinical Sciences, Skåne University Hospital, Malmö, Sweden; ²Department of Rehabilitation Medicine, Skåne University Hospital, Malmö, Sweden and ${ }^{3}$ Department of Health Sciences, Lund University, Lund, Sweden

Correspondence: Dr S Acosta, Vascular Center, Institution for Clinical Sciences, Skåne University Hospital, 20502 Malmö, Sweden.

E-mail: Stefan.acosta@telia.com

Received 10 February 2014; revised 5 August 2014; accepted 12 August 2014; published online 23 September 2014 
twelve patients were diagnosed with permanent and transient SCI, respectively. Patients were followed for a median time of 26 months (range 14-56) from day of operation, and information on survival was retrieved from the Swedish Population Registry on 5 September, 2013. Mid-term follow-up refers to evaluation of outcome in patients, $2-5$ years after complex and emergent complex endovascular aneurysm repair procedures. ${ }^{8}$

Telephone contact was taken with the remaining 21 surviving patients. Three patients had no neurological deficits and were excluded from the study. The remaining 18 patients reported symptoms indicating neurological deficits related to SCI and were offered a home visit for an $\sim 1$-hour-long interview regarding medical aspects, everyday life aspects and life satisfaction (Figure 1). Interviews were carried out throughout September 2013 at the patients' homes $(n=18)$. None of the patients lived in a nursing home.

\section{At-home interview}

Information about need of home-care services, physiotherapy and primary use of mobility aids (that is, a cane, a crutch or two crutches, a walking frame, or a wheelchair) was obtained during the interview. Home-care services correspond to the Swedish home-care services meaning that medically trained personnel visited the patient at home and helped with the activities of daily living (ADL). Urinary dysfunction postoperatively was defined as need of indwelling catheter or clean intermittent catheterization, or having new onset of symptoms indicating neurogenic bladder. Urge incontinence was defined as new onset of involuntary leakage of urine and a sudden urge to urinate. Bowel dysfunction was defined as stoma today or planned, obstipation or incontinence. The Spinal Cord Independence Measure, ${ }^{9}$ a disability scale for patients with spinal cord lesions, which assesses independence in daily activities, was used to define obstipation: irregular timing or very low frequency (less than once in 3 days) of bowel movements. Obstipation was defined as manageable with the help of laxatives or unmanageable. Sexual dysfunction was defined as inability or reduced ability to perform the sexual act. No or reduced genital sensation was registered. Spasticity in the legs, current decubitus ulcer and prescribed medications were documented.

\section{Life satisfaction}

Life satisfaction was assessed with two self-report questionnaires; the Life Satisfaction Questionnaire (LiSat-11) and the Satisfaction With Life Scale (SWLS). The LiSat- $11^{10}$ contains 11 items rated on a six-point Likert scale ( $1=$ very dissatisfied to $6=$ very satisfied). It includes one item assessing global life satisfaction ('Life as a whole') ${ }^{10}$ and 10 domain-specific items. The resulting scores were dichotomized in 1-4 (not satisfied) and 5-6 (satisfied) for comparison with a Swedish reference sample. ${ }^{10}$ SWLS ${ }^{11}$ comprises five items rated on a seven-point Likert scale $(1=$ strongly disagree to $7=$ strongly agree $)$. Total scores range from 5 to 35 , with higher scores reflecting greater life satisfaction. To compare the results (seven-point scale) with the reference sample (five-point scale), scores were recoded as $1=1 ; 2$ and $3=2 ; 4=3 ; 5$ and $6=4$; and $7=5$ as seen in a previous study. ${ }^{12,13}$

\section{Neurological deficits}

Neurological deficits, T1-S5, were graded according to the American Spinal Injury Association (ASIA) impairment scale (AIS) in patients discharged with permanent SCI from hospital: AIS A, complete, no motor or sensory function is preserved in the sacral segments S4-S5; AIS B, incomplete, sensory but not motor function is preserved below the neurological level and includes the sacral segments S4-S5; AIS C, incomplete, motor function is preserved below the neurological level, and more than half of key muscles below the neurological level have a muscle grade $<3$; AIS D, incomplete, motor function is preserved below the neurological level, and at least half of key muscles below the neurological level have a muscle grade of 3 or more. The neurological deficits in each patient were re-scrutinized and re-assessed after evaluation of the respective patient records by a specialist in neurology. Any results of urodynamic investigation were noted.

\section{Statistics}

SPSS software (version 20.0; SPSS Inc, Chicago, IL, USA) was used for data managing and statistical analysis. Continuous variables were expressed as median and range or interquartile range, or mean and s.d. Proportions were expressed in percentage with $95 \%$ confidence interval modified by using the Wald method. ${ }^{14}$ Confidence interval for the difference in proportions between the studied and reference population ${ }^{10}$ was calculated. Mann-Whitney $U$-test was used for asymmetric continuous data in group comparisons. Fisher's exact test was used for cross-sectional, categorical values in group comparisons. Student's $t$-test was used when comparison of means and s.d. was made between the studied and reference sample, ${ }^{12,13}$ where alpha level was set at 0.01 and variance at equal, at the website: in-silico.net/tools/statistics/test; the 17 December 2013. $P<0.05$ was considered as significant.

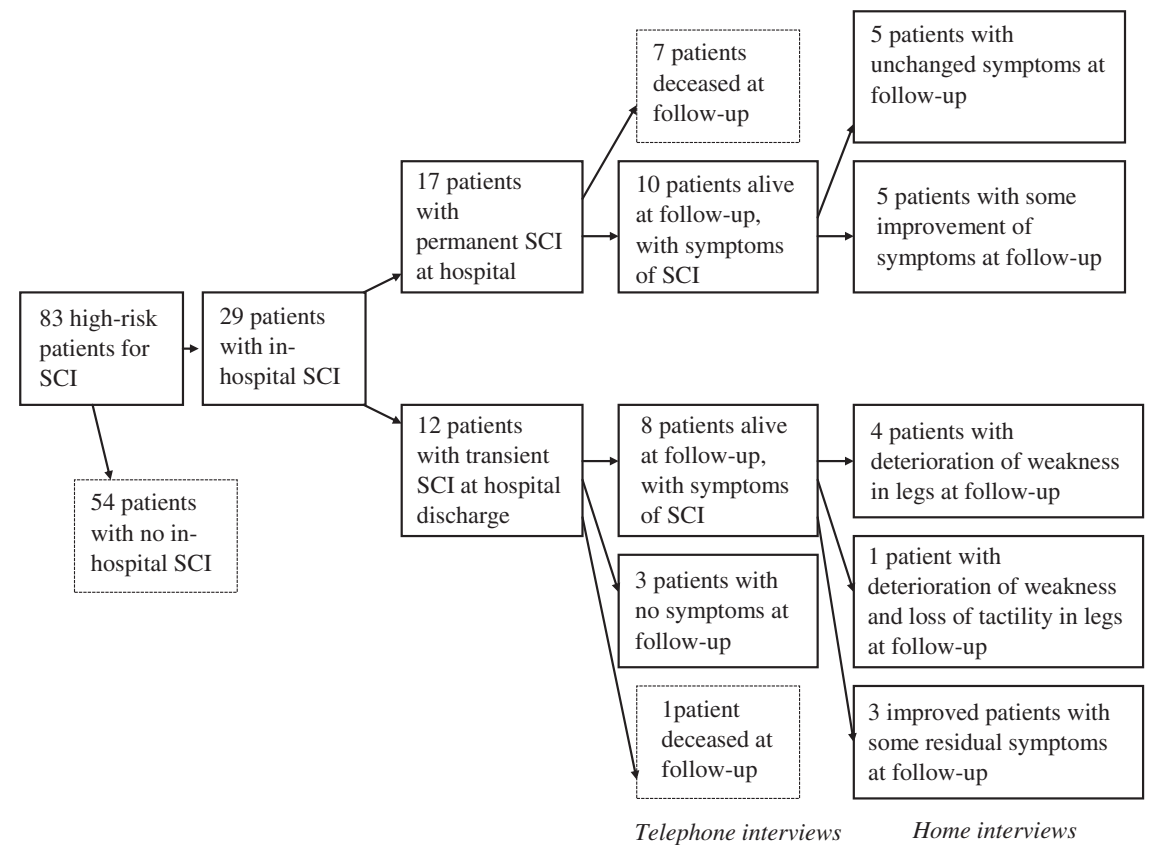

Figure 1 Flowchart of patients with spinal cord ischemia after TAEVAR. 


\section{RESULTS}

\section{Patient characteristics}

Pre-operative patient characteristics are shown in Table 1. The patients had the following aortic diseases: thoracic aortic aneurysm $(n=3$, $17 \%)$, thoraco-abdominal aortic aneurysm $(n=12,67 \%)$, juxta-renal aortic aneurysm $(n=2,11 \%)$ and infra renal aortic aneurysm $(n=1$, $6 \%$ ). The thoraco-abdominal aortic aneurysm was classified as type 1 $(n=3)$, type $2(n=6)$, type $3(n=2)$ and type $4(n=1)$. Indications for therapy were aortic aneurysm $(n=17,94 \%)$ and pseudo-aneurysm $(n=1,6 \%)$. Stent grafts were positioned in the thoracal $(n=3,17 \%)$, thoraco-abdominal $(n=14,78 \%)$ and abdominal $(n=1,6 \%)$ part of the aorta. At hospital discharge, 10 and 8 patients were judged as having a permanent or transient SCI, respectively (Figure 1). Three underwent acute operation, all had transient SCI. Fifteen underwent elective operation. At follow-up, mortality in patients with permanent SCI (7/17) was higher than those with transient SCI $(1 / 12)$ $(P=0.035)$.

\section{Cerebrovascular disease}

One patient with permanent SCI suffered from a stroke 3 months preoperatively. Two patients with transient SCI suffered from stroke; one patient had several transient ischemic attacks preoperatively and the other one had a perioperative cerebral infarction.

\section{The anatomical location of SCI}

Fourteen patients were clinically assessed in-hospital by a consulting neurologist from a timespan of the same day until 13 days after debut of SCI symptoms: ten had the anterior spinalis syndrome, one had symptoms of spinal shock and two assessments were inconclusive.

\section{Neurological deficits during follow-up}

Ten patients had permanent T1-S5 SCI, two were classified as AIS A, one as AIS B and seven as AIS D. In total, two patients had complete and eight had incomplete SCI. Nine were discharged to a rehabilitation center, and one patient was sent home due to improvement of the SCI related symptoms. Eight patients had transient SCI at hospital discharge, and they all expressed concerns at follow-up regarding the function of their legs (Figure 1).

Table 1 Preoperative characteristics of patients undergoing TAEVAR and who postoperatively developed spinal cord ischemia

Total $(\mathrm{n}=18)$

(\%)

\begin{tabular}{lc} 
Age at injury (years) & 70 (range 54-76) \\
Sex (Male/Female) & $15(83) / 3(17)$ \\
Follow-up (months) & 26 (range 14-56) \\
History of: & $18(100)$ \\
Hypertension & $16(89)$ \\
Smoking & $3(17)$ \\
Diabetes mellitus & $8(44)$ \\
Ischemic heart disease & $2(11)$ \\
Stroke or transient ischemic attack & $7(39)$ \\
COPD & $13(72)$ \\
Renal function impairment & $4(22)$ \\
Cancer diagnosis & \\
Medication & $14(78)$ \\
Lipid-lowering agent & $15(83)$ \\
Acetyl salicylic acid & \\
\hline
\end{tabular}

\section{Mobility at follow-up}

Patients with permanent SCI at hospital discharge were to a greater extent wheelchair-dependent $(P=0.025)$ compared with the patients with transient SCI at discharge (Table 2).

\section{Investigation and treatment of bladder malfunction at follow-up} Cystometry was performed in five patients, and all were diagnosed with neurogenic bladder dysfunction. At follow-up, patients with permanent SCI at hospital discharge had significantly higher proportion of urinary $(P=0.007)$ dysfunction than the patients with transient SCI (Table 2). Three patients with permanent SCI used clean intermittent self-catheterization and three used an indwelling catheter. In all, three patients received anti-cholinergic treatment against urinary urge incontinence.

\section{Bowel dysfunction at follow-up}

Five patients, all with permanent SCI, had fecal incontinence inhospital and were clinically diagnosed with neurogenic bowel dysfunction. At follow-up, patients with permanent SCI had significantly higher proportion of bowel dysfunction $(P=0.043)$ compared with patients with transient SCI (Table 2).

\section{Sexual dysfunction at follow-up}

New onset of sexual dysfunction after TAEVAR was found in 11 out of 14 patients at follow-up (Table 2). Among the ten patients with permanent SCI, six men reported inability and two men reported reduced ability to perform a sexual act. Four reported no sensation and the other four reported reduced sensation in the genital area. Among the eight patients with transient SCI, two men reported reduced ability to perform a sexual act, where one had no sensation and the other had reduced sensation in the genital area.

\section{Life satisfaction at follow-up}

LiSat-11. Patients with SCI in the study were found to have a lower self-rated life satisfaction compared with the reference group in terms of 'life as a whole', 'sexual life', 'somatic health' and psychological health', but showed a higher satisfaction with the 'economy' domain (Table 3).

SWLS. The study sample was found to have a lower self-rated life satisfaction on three of five items in the SWLS questionnaire (SWLS 1-3), 'in most ways my life is close to my ideal', 'the conditions of my life are excellent' and 'I am satisfied with my life', compared with the reference sample (Table 4).

\section{DISCUSSION}

The main finding of this study was that the majority of patients with SCI in-hospital who were discharged with apparent full recovery of neurological deficits, the transient group, in fact did have minor selfreported neurological sequel at follow-up. Hence, a neurological follow-up post discharge of all patients with in-hospital SCI, whether with permanent or transient symptoms, seems to be mandatory to identify all patients with neurological deficits and to be able to provide proper follow-up investigations such as urodynamic studies and assessment of functional ability.

The neurological deficits at follow-up seem all, apart from the preand perioperative stroke events in three patients, to be attributed to the SCI as a complication after the endovascular procedure. The predominant neurological deficits were attributed to loss of motor function and the corresponding lesions located to the anterior cord, a 
Table 2 Findings after home interviews in relation to whether the patients were discharged with permanent or transient spinal cord ischemia at discharge from hospital after TAEVAR and having neurological deficits at follow-up

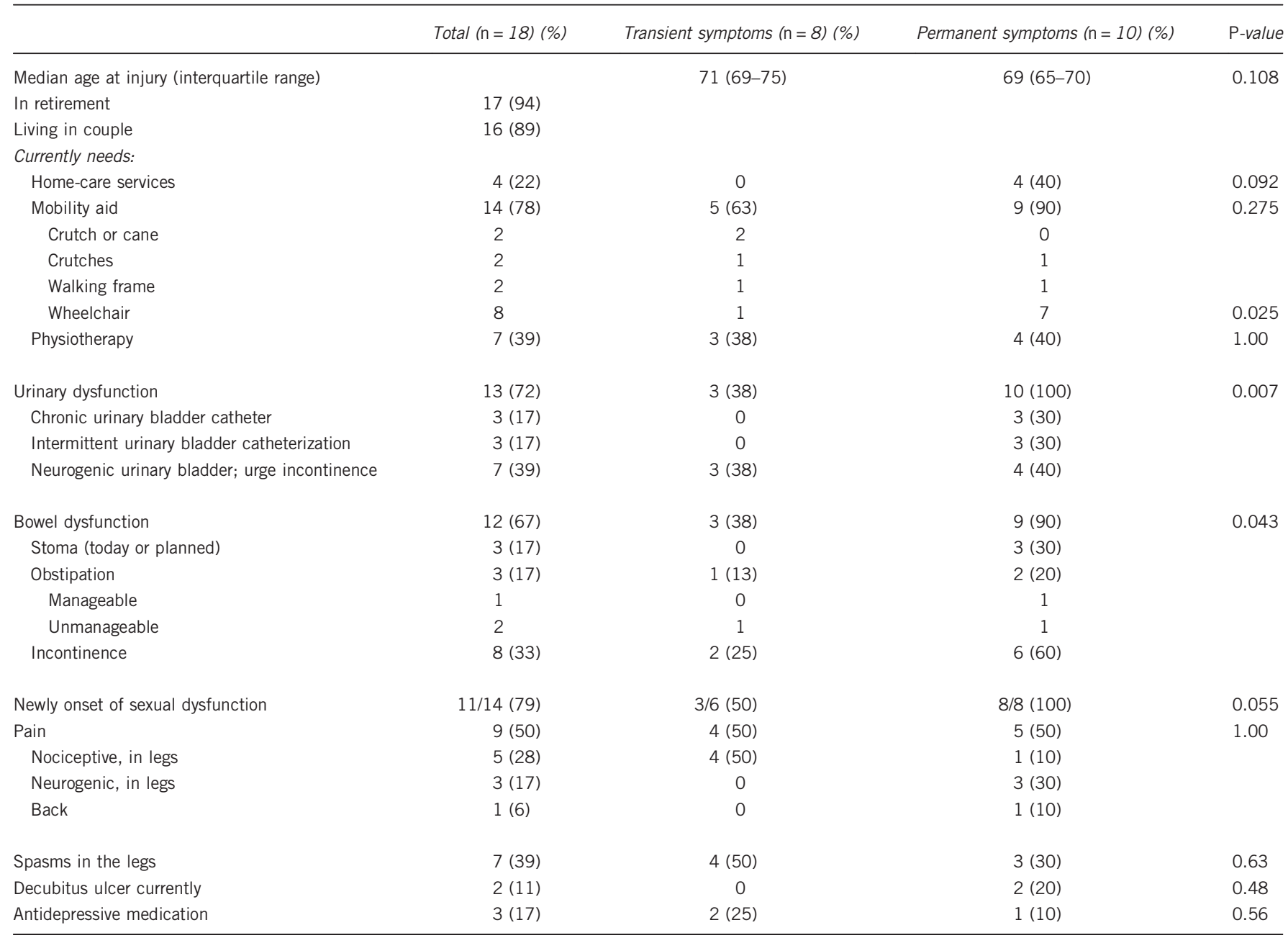

Table 3 Comparison of self-reported levels of life satisfaction (LiSat-11) in patients with spinal cord ischemia after TAEVAR and the Swedish reference sample

\begin{tabular}{lccc}
\hline & $\begin{array}{c}\text { Patients with spinal cord injury }(\mathrm{n}=18 ; \\
(95 \% \mathrm{Cl}))\end{array}$ & $\begin{array}{c}\text { Swedish population (Fugl-Meyer et al., }{ }^{10} \\
\mathrm{n}=2533,(95 \% \text { Cl) }\end{array}$ & $\begin{array}{c}\text { Confidence interval for the difference of two } \\
\text { population proportions }\end{array}$ \\
\hline Life as a whole & $44(0.25-0.66)$ & $70(0.68-0.72)$ & $0.030-0.49^{\mathrm{a}}$ \\
Vocation & $50(0.29-0.71)$ & $54(0.52-0.56)$ & $(-0.19)-0.27$ \\
Economy & $67(0.44-0.84)$ & $39(0.37-0.41)$ & $(-0.062)-(-0.50)^{\mathrm{a}}$ \\
Leisure & $39(0.20-0.61)$ & $57(0.55-0.59)$ & $(-0.046)-0.41$ \\
Contacts with friends & $61(0.39-0.80)$ & $65(0.63-0.67)$ & $(-0.19)-0.27$ \\
Sexual life $(n=17)$ & $12(0.019-0.34)$ & $82(0.80-0.83)$ & $0.54-0.86^{\mathrm{a}}$ \\
Activities of daily life & $44(0.25-0.66)$ & $56(0.54-0.58)$ & $(-0.11)-0.35$ \\
Family life $(n=17)$ & $88(0.66-0.98)$ & $81(0.79-0.82)$ & $(-0.23)-0.085$ \\
Partner relationship $(n=16)$ & $94(0.72-1.0)$ & $95(0.94-0.96)$ & $(-0.11)-0.13$ \\
Somatic health & $22(0.085-0.46)$ & $72(0.70-0.74)$ & $0.31-0.69^{a}$ \\
Psychological health & $56(0.34-0.75)$ & $81(0.79-0.82)$ & $0.020-0.48^{\mathrm{a}}$ \\
\hline
\end{tabular}

aDifference between the compared groups.

common clinical feature after acute occlusion of the dominant spinal artery, Adamkiewicz. Moreover, one patient had a cerebral infarction peri-operatively, which is within the reported stroke incidence range from 4.1 to $7.6 \% .^{15}$
This study showed a high frequency of neurogenic bladder among the studied patients, even if relatively few cystometries were performed. It seems that the urinary problems were neglected by the health-care providers. The results suggest that all patients with a SCI, 
Table 4 The satisfaction with life scale (SWLS) for 18 patients with spinal cord ischemia after TAEVAR

\begin{tabular}{|c|c|c|c|c|}
\hline & \multirow{2}{*}{$\begin{array}{l}\text { Seven-point scale }(\mathrm{n}=18 ; \\
\text { mean; (s.d.; range)) }\end{array}$} & \multicolumn{3}{|c|}{ Five-point scale } \\
\hline & & $\begin{array}{l}\text { Spinal ischemia sample } \\
\text { (mean; (s.d.)) }\end{array}$ & $\begin{array}{c}\text { Reference sample } \\
(\mathrm{n}=2900 ; \text { mean; }(\text { s.d. }))\end{array}$ & P-value \\
\hline $\begin{array}{l}\text { SWLS 1: in most ways my life } \\
\text { is close to my ideal }\end{array}$ & $3.94(2.39 ; 1-7)$ & $3.00(1.50)$ & $3.98(0.80)$ & $P<0.0001$ \\
\hline $\begin{array}{l}\text { SWLS 3: I am satisfied with } \\
\text { my life }\end{array}$ & $4.56(2.12 ; 1-7)$ & $3.33(1.37)$ & $4.21(0.77)$ & $P<0.0001$ \\
\hline $\begin{array}{l}\text { SWLS 4: so far I have gotten the important } \\
\text { things I want in life }\end{array}$ & $5.11(1.61 ; 1-7)$ & $3.78(1.00)$ & $3.90(0.90)$ & $P=0.57$ \\
\hline $\begin{array}{l}\text { SWLS 5: if I could live my life over, I would } \\
\text { change almost nothing }\end{array}$ & $5.06(1.96 ; 1-7)$ & $3.67(1.24)$ & $3.67(1.03)$ & $P=1.00$ \\
\hline
\end{tabular}

whether with transient or permanent symptoms, ${ }^{16-18}$ should be investigated for neurogenic bladder at follow-up. A cystometry should be performed to confirm or exclude the condition, to evaluate whether urinary retention is present and medication for neurogenic bladder should be considered.

Some of the patients with SCI had severe symptoms of bowel dysfunction, necessitating a better control of bowel emptying. The two wheelchair-dependent patients had a controlled output colostoma, and one patient was planned for colostomy due to incontinence for stools. Patients with neurogenic bowel may have many severe deficits such as weak abdominal muscles, impaired rectal sensation and delayed colonic transit time resulting in obstructed defecation, whereas impaired external anal sphincter contraction and uninhibited rectal contractions result in incontinence for stools. ${ }^{19}$ It is hard to tell if anticholinergic treatment for urge incontinence or treatments with laxatives in some patients might have worsened the patients' bowel function. A large proportion of patients were bothered by bowel dysfunction, suggesting the importance of a thorough evaluation of the patients' discomforts at follow-up.

The reports and the life satisfaction questionnaires showed a very low satisfaction with sexual life after the operation. This finding was, however, expected. ${ }^{20,21}$ The patients need to be informed of this, often neglected, complication preoperatively, and they should be asked postoperatively about their sexual function, to enable a possible treatment.

Compared with the Swedish reference samples, the studied population with predominantly males showed lower life satisfaction scores, despite that the partner relationship rate, a factor strongly associated with improved life satisfaction scores, ${ }^{10}$ was very high, $89 \%$. A previous report has shown that it is most important to have a nation-specific reference group, as cultural differences may have an impact on life satisfaction scores. ${ }^{22}$ The reference groups comprised Swedish populations between the ages of 18-64 years ${ }^{10}$ and $27-71$ years, ${ }^{13}$ respectively. Hence, these control groups were younger but equal in gender distribution. Life satisfaction scores seem neither to be gender dependent, nor to be associated with age. ${ }^{23}$ In two studies among older adults, level of life satisfaction was found to be the same across all ages. ${ }^{24,25}$ Furthermore, older age has been seen to be positively associated with family life and financial and vocational situation. ${ }^{23}$ In contrast, other studies have suggested caution when comparing life satisfaction with different age groups; ${ }^{23}$ however, this uncertainty might be due to SWLS items $4-5$, which have been shown to be sensitive to age, ${ }^{12}$ whereas SWLS items $1-3$ have been found to be insensitive to age. Thus, the finding of a lower life satisfaction according to SWLS scores for items 1-3 among the studied patients seems quite reasonable. Surprisingly, patients with SCI were found to have a higher level of satisfaction with their economy compared with the reference group. This finding might reflect the fact that the majority of the study patients lived in a couple relationship, hence obtaining a double income. It might also reflect the good social standard in Sweden.

The present study would have been strengthened if another more age, gender and co-morbidity matched control group was added such as patients undergoing TAEVAR without suffering from SCI during the same time period. However, there may be concerns of feasibility to create such a control group and, above all, the number of patients would always be greatly under-sized compared with the used reference samples in the present study. In a more age, gender and co-morbidity matched historical control group of patients who had undergone open repair for abdominal aortic aneurysm without suffering from SCI, life satisfaction evaluation by LiSat-11 showed that sexual functioning and psychological health were the domains that were most suppressed, whereas the other domains appeared to be very little affected. ${ }^{26}$ In the present study, there were three patients suffering from pre- and perioperative stroke, which could have influenced their life satisfaction. Indeed, life satisfaction scores have been shown to be significantly impaired in stroke survivors compared with healthy controls. ${ }^{27}$ Nevertheless, the present study suggests that the low life satisfaction scores in patients treated with advanced endovascular therapy for thoraco-abdominal aortic aneurysm disease were related to the occurrence of permanent SCI with its profound impact on limb, bowel, urinary and sexual functioning. Patients with SCI represent a small group of patients with a disastrous complication after advanced endovascular therapy for aortic disease, with increased mortality rate, ${ }^{1}$ and the present study supports previous reported findings that patients with permanent SCI at discharge have a worse prognosis than patients with transient SCI. ${ }^{1}$ For natural reasons, vascular surgeons avoid reporting on their most severe complications and very few publications are therefore available. No prospective studies exist. Therefore, the present mid-term follow-up investigation with home interviews assessing functional ability and life satisfaction contributes greatly to the accumulated body of knowledge in these SCI patients. Despite the reduced level of life satisfaction among the study patients at follow-up, some of these patients were thankful for having been treated for their potentially lethal aortic disease. 
In conclusion, the assessment of life satisfaction at mid-term followup suggests that all patients with SCI in-hospital, whether permanent or transient, should have a multi-disciplinary follow-up regime. Most patients diagnosed with transient neurological deficits had an overlooked permanent, less severe, SCI.

\section{DATA ARCHIVING}

There were no data to deposit.

\section{CONFLICT OF INTEREST}

The authors declare no conflict of interest.

\section{ACKNOWLEDGEMENTS}

We thank Magnus Wahlqvist, Department of Neurology, Skåne University hospital, Malmö, for re-assessing extent and severity of neurological deficits.

1 DeSart K, Scali ST, Feezor RJ, Hong M, Hess PJ Jr., Beaver TM et al. Fate of patients with spinal cord ischemia complicating thoracic endovascular aortic repair. J Vasc Surg 2013; 58: 635-642.

2 Ullery B, Cheung A, Fairman R, Jackson B, Woo E, Bavaria J et al. Risk factors, outcomes and clinical manifestations of spinal cord ischemia following thoracic endovascular aortic repair. J Vasc Surg 2011; 54: 677-684.

3 Lee AL, Daniels MJ, Beaver TM, Klodell CT, Raghinaro DE, Hess PJ. Late outcomes of a single-center experience of 400 consecutive thoracic endovascular aortic repairs. Circulation 2011; 123: 2938-2945.

4 Estrera AL, Miller CC, Huynh TT, Porat E, Safi HJ. Neurologic outcome after thoracic and thoracoabdominal aortic aneurysm repair. Ann Thorac Surg 2001; 72 1225-1230.

5 Lederle FA, Johnson GR, Wilson SE, Ballard DJ, Jordan WD Jr., Blebea J et al. Rupture rate of large abdominal aortic aneurysms in patients refusing or unfit for elective repair. JAMA 2002; 287: 2968-2972.

6 Kirshblum SC, Burns SP, Biering-Sorensen F, Donovan W, Graves DE, Jha A et al. International standards for neurological classification of spinal cord injury (revised 2011). J Spinal Cord Med 2011; 34: 535-546.

7 Mehmedagic I, Resch T, Acosta S. Complications to cerebrospinal fluid drainage and predictors of spinal cord ischemia in patients with aortic disease undergoing advanced endovascular therapy. Vasc Endovasc Surg 2013; 47: 415-422.

8 Boyle J, Thompson M, Vallabhaneni R, Bell R, Brennan J, Browne T et al. For the British Society of Endovascular Therapy. Pragmatic minimum reporting standards for endovascular abdominal aortic aneurysm repair. J Endovasc Ther 2011; 18: 263-271.

9 Catz A, Itzkovich M, Agranov E, Ring H, Tamir A. SCIM-spinal cord independence measure: a new disability scale for patients with spinal cord lesions. Spinal cord 1997 35: 850-856.
10 Fugl-Meyer AR, Melin R, Fugl-Meyer KS. Life satisfaction in 18- to 64-year-old Swedes: in relation to gender, age, partner and immigrant status. Eur Board of Phys Rehab Med 2002; 34: 239-246.

11 Diener E, Emmons RA, Larsen RJ, Griffin S. The satisfaction with life scale. J Pers Assess 1985; 49: 71-75.

12 Hultell D, Gustavsson JP. A psychometric evaluation of the Satisfaction With Life Scale in a Swedish nationwide sample of university students. Pers Individ Diff 2008; 44: 1070-1079.

13 Jacobsson LJ, Westerberg M, Lexell J. Health-related quality-of-life and life satisfaction 6-15 years after traumatic brain injuries in northern Sweden. Brain Injury 2010; 24: 1075-1086.

14 Agresti A, Coull B. Approximate is better than "exact" for interval estimation of binomial proportions. Am Stat 1998; 52: 119-126.

15 Cheng D, Martin J, Shennib H, Dunning J, Muneretto C, Schueler S et al. Endovascular aortic repair versus open surgical repair for descending thoracic aortic disease a systematic review and meta-analysis of comparative studies. J Am Coll Cardiol 2010; 55: 986-1001.

16 Akkoc Y, Cinar Y, Kismali E. Should complete and incomplete spinal cord injury patients receive the same attention in urodynamic evaluations and ultrasonography examinations of the upper urinary tract? Int J Rehab Res 2012; 35: 178-180.

17 Moslavac S, Dzidic I, Kejla Z. Neurogenic detrusor overactivity: comparison between complete and incomplete spinal cord injury patients. Neurouro Urodyn 2008; 27: 504-506.

18 Gomelsky A, Lemack GE, Weld KJ, Dmochowski RR. Urodynamic patterns following ischemic spinal cord events. J Urol 2003; 170: 122-125.

19 Valles M, Mearin F. Pathophysiology of bowel dysfunction in patients with moto incomplete spinal cord injury: comparison with patients with motor complete spinal cord injury. Dis Col Rectum 2009; 52: 1589-1597.

20 Biering-Sorensen F, Sonksen J. Sexual function in spinal cord lesioned men. Spinal cord 2001; 39: 455-470.

21 Kreuter M, Taft C, Siosteen A, Biering-Sorensen F. Women's sexual functioning and sex life after spinal cord injury. Spinal cord 2011; 49: 154-160.

22 Ide M, Fugl-Meyer AR. Life satisfaction in persons with spinal cord injury: comparative investigation between Sweden and Japan. Spinal Cord 2001; 39 : 387-393.

23 Clench-Aas J, Nes RB, Dalgard OS, Aaro LE. Dimensionality and measurement invariance in the Satisfaction with Life Scale in Norway. Qual Life Res 2011; 20 : 1307-1317.

24 Hamarat E, Thompson D, Aysan F, Steele D, Matheny K, Simons C. Age differences in coping resources and satisfaction with life among middle-aged, young-old, and oldest old adults. J Genet Psychol 2002; 163: 360-367.

25 Siedlecki KL, Tucker-Drob EM, Oishi S, Salthouse TA. Life satisfaction across adulthood: different determinants at different ages? J Pos Psych 2008; 3: 153-164.

26 Hallin A, Bergqvist D, Fugl-Meyer K, Holmberg L. Areas of concern, quality of life and life satisfaction in patients with peripheral vascular disease. Eur J Vasc Endovasc Surg 2002; 24: 255-263.

27 Laurent K, De Seze MP, Delleci C, Koleck M, Dehail P, Orgogozo JM et al. Assessment of quality of life in stroke patients with hemiplegia. Ann Phys Rehabil Med 2011; 54: 376-390. 